\title{
Life, Physical, and Social Science Occupations
}

National Cancer Institute

\section{Source}

National Cancer Institute. Life, Physical, and Social Science Occupations. NCI Thesaurus.

Code $C 97638$.

A class of professional or vocational positions of employment that involve life, physical or social sciences. 